\title{
REVIEW
}

\section{Changes in metabolic syndrome parameters after liposuction}

\author{
Payer J Jr${ }^{1}$, Ziak $\mathrm{P}^{1}$, Fedeles $\mathrm{J} \mathrm{Jr}^{1}$, Brazdilova $\mathrm{K}^{2}$, Fedeles $\mathrm{J}^{1}$ \\ Department of Plastic Surgery, Medical Faculty of Comenius University and University Hospital Bratislava, \\ Bratislava,Slovakia.juraj.payer@gmail.com
}

\begin{abstract}
Liposuction - one of the most frequent procedures in cosmetic surgery - is the surgical aspiration of fat from the subcutaneous layer leaving a more desirable body contour and leaving a smooth transition between the suctioned and nonsuctioned areas. Metabolic syndrome is epidemiologically important medical condition that includes insulin resistance, dyslipidemia, central obesity, hypertension, impaired glucose tolerance or diabetes mellitus, and high rates of atherosclerotic disease. According to the latest findings, liposuction reduces the amount of subcutaneous fat, which changes the abdominal - superficial adipose tissue ratio and thus might affect the potential of metabolic syndrome by the means of its separate parameters and clinical manifestations. Results that can be found in the published literature remain controversial and often contradictory, thus leaving enough space for further investigations regarding the relation of these two clinical entities (Ref. 33). Full Text in PDF www.elis.sk.

Key words: liposuction, metabolic syndrome, adipose tissue.
\end{abstract}

Liposuction is the surgical aspiration of fat from the subcutaneous layer leaving a more desirable body contour and leaving a smooth transition between the suctioned and nonsuctioned areas (1). It is the most commonly performed aesthetic surgical procedure in the United States (2).

In 1988, Reaven proposed that insulin resistance is central to the aetiology of type 2 diabetes mellitus, hypertension, and coronary artery disease (3). The concept of insulin resistance and associated metabolic abnormalities leading to an increased risk of cardiovascular disease became known by a variety of names, including metabolic syndrome, dysmetabolic syndrome, syndrome $\mathrm{X}$, cardiometabolic syndrome, and insulin resistance syndrome (4).

Clinical findings most commonly associated with metabolic syndrome include insulin resistance, dyslipidemia (specifically high triglycerides, low levels of high-density lipoprotein (HDL), and small dense low-density lipoprotein (LDL)), central obesity, hypertension, impaired glucose tolerance or diabetes mellitus, and high rates of atherosclerotic disease. Recently, markers of inflammation and accelerated hemostasis / impaired fibrinolysis have been added (4).

Though nowadays many authors argue if the abovementioned combination of clinical findings and symptoms really forms a true syndrome, there is no doubt in their close relation and frequent coincidence.

${ }^{1}$ Department of Plastic Surgery, Medical Faculty of Comenius University and University Hospital Bratislava, Bratislava, Slovakia, and ${ }^{2} 5$ th Department of Internal Medicine, Medical Faculty of Comenius University and University Hospital Bratislava, Bratislava, Slovakia

Address for correspondence: J. Payer, Jr, MD, Department of Plastic Surgery, Medical Faculty of Comenius University and University Hospital Bratislava, Ruzinovska 6, SK-826 06 Bratislava, Slovakia.
Liposuction reduces the amount of subcutaneous fat, which changes the abdominal - superficial adipose tissue ratio and thus might affect the potential of metabolic syndrome by the means of it is separate parameters and clinical manifestations.

\section{Lipid metabolism}

The effect of liposuction on plasma cholesterol levels is highly controversial. The results are ambiguous. They differ from author to author showing both positive and negative, or, in some cases, even no effect.

\section{Cholesterol}

Peroperative LDL-cholesterol levels elevation in a regular volume liposuction (around $1500 \mathrm{ml}$ of lipoaspirate) have been described, but they drop immediately postoperatively and reach their initial values already 4 hours after the procedure (5). It has been proven that in non-obese women plasma LDL-cholesterol levels are significantly lower 3 weeks as well as $2-4$ months postoperatively than before a liposuction $(6,7)$, while other studies showed an opposite effect and a significant increase in LDL 6 months after a regular volume liposuction in women with the average BMI of $26,17 \mathrm{~kg} / \mathrm{m}^{2}(8)$. Swanson didn’t observe any effect of liposuction on LDL, HDL or overall blood cholesterol levels 3 months post liposuction in his recent prospective study of 322 patients (9). Similar results have been described by others as well, showing in obese women no effect of large-volume liposuction (more than $5000 \mathrm{ml}$ of lipoaspirate) on cholesterol levels $2-3$ months postoperatively $(10,11)$. Cazes and colleagues observed a drop in LDL and overall cholesterol levels 3 months after liposuction in obese patients, but the values returned to baseline 1 year postoperatively (12). In Hong's study of 11 orientals, the $5.1 \%$ average decrease of serum LDL-cholesterol levels has been described (13). Pedroza 
observed a cholesterolemia decrease post liposuction in 22 originally hypercholesterolemic patients, reaching the standard normal levels during a 5-year follow-up. The subjects were advised to hang on to a low-fat diet and exercise regularly 2-3 times a week (14).

Ybarra and colleagues found out that cholesterol levels were significantly affected by liposuction showing the average $10 \%$ HDL increase and $15.2 \%$ VLDL decrease 4 months postoperatively (15). A significant HDL-cholesterol increase in obese patients has even been described 6 to 12 months post large-volume liposuction $(16,17)$. A sooner effect of LVL on HDL is absent 6 weeks to 4 months postoperatively (18). Orientals even showed a slight (3.8\% in average) decrease of HDL levels 2 months after LVL (11).

\section{Triglycerides and free fatty acids}

When undergoing a regular volume liposuction, the patients present with an intraoperative increase in triglyceride levels, but the values return to baseline only 4 hours postoperatively (5). Other authors described a decrease in plasma triglycerides already during liposuction persisting for the first 7 postoperative days (14). Some papers showed a significant, up to $35 \%$, decrease in free fatty acid levels and $21.3 \%$ drop in triglycerides 4 months after liposuction (15). Mohammed and colleagues didn't prove any long-term effect of abdominal large-volume liposuction on triglyceride or HDL and LDL-cholesterol levels in 7 obese females (19).

The plasma triglyceride, as well as LDL levels, decrease has been demonstrated in a study following originally hypercholesterolemic patients 4-12 months after liposuction (20). A similar drop in triglyceride values reaching almost $25 \%$ in average has been observed in a cohort of 205 obese patients 8 weeks after liposuction (21) as well as in the recently published prospective study of Swanson, where the levels dropped by $43 \%$ in average 3 months postoperatively in 56 preoperatively hypertriglyceridemic patients (9).

\section{Subcutaneous to visceral fat ratio, waist circumference}

Suctioning subcutaneous fat in large-volume liposuction causes an immediate increase of the visceral to subcutaneous fat ratio by up to $12 \%$ in women and almost $14 \%$ in men, which may lead to a higher risk of metabolic complications of obesity (22). Other authors share the same opinion when describing no effect of liposuction, and the accompanying weight reduction, on reducing visceral fat volume in a 6 months follow-up of overweight patients (8).

However, a redistribution of subcutaneous fatty tissue occurs postoperatively and a year after undergoing liposuction the subcutaneous fat layer of non-obese patients after small-volume liposuction reaches comparable volume to the numbers obtained from the control group individuals, that didn't undergo any procedure, in DXA and MRI measurements. Though a subcutaneous fat reduction retains its long-term effect in the hips area (23). In the recently published trial by Benatti and colleagues, abdominal liposuction did not induce regrowth of fat, but it triggered a compensatory increase of visceral fat unless this was effectively counteracted by physical activity (24).
Klein has shown opposite results when observing a significant decrease of abdominal fat volume 3 months post abdominal largevolume liposuction (10). According to Ybarra, the waist circumference decreased significantly by $5.9 \%$ in average in normal or overweight individuals (15). Other authors reported a significant improvement of the waist : hips ratio in obese patients 3 weeks as well as 3 months post LVL (25).

\section{Blood glucose levels and insulin resistance}

According to older studies, fasting glucose remains unaffected 9-12 months post LVL in obese patients (16) and it even stays unchanged 2 or more years postoperatively despite the long-term effect on body fat volume reduction (17). However, a case of reducing blood fasting glucose levels from values over $11 \mathrm{mmol} / \mathrm{l}$ to normal levels one year after LVL has been reported in a patient who continued diet and weight reduction postoperatively (26). Latest studies even showed the average of $18 \%$ fasting glucose and $2.3 \%$ plasma glycated hemoglobin (HbA1C) levels reduction in obese patients one year after undergoing LVL (27).

Insulin sensitivity is significantly higher in obese patients one month post LVL maintaining the improved level for the 2 following months (25) and according to Giugliano's study, there was a significant decrease of insulin resistance assessed by the means of Homeostasis Model Assessment (HOMA = (fasting glucose $\mathrm{x}$ insulinemia)/25) together with plasma adiponectin elevation even 6 months postoperatively (17). Lowering of fasting plasma insulin and improved insulin sensitivity has been described as long as 4 months post liposuction $(7,18)$, or generally after any kind of subcutaneous lipectomy with or without excising excess skin (28). When combined with diet, liposuction significantly improved insulin sensitivity even when compared to "diet-only" control, where the patients didn't undergo any kind of surgery (29).

However, the effect of liposuction on insulin levels and insulin sensitivity was not found in non-obese patients (6). Blood glucose levels or insulinemia didn't respond to liposuction in normal weight or slightly overweight patients $(9,15)$. Insulin sensitivity remains also unchanged 3 months post LVL in both groups in the study comparing otherwise healthy obese women to obese patients suffering from type II diabetes (10).

\section{Arterial blood pressure}

We may find two contradictory types of results when we search for the relation of liposuction and arterial blood pressure in the available published papers.

According to some authors, there is a significant lowering of systolic arterial blood pressure by more than $10 \mathrm{mmHg} 4$ months after LVL in females with BMI 25-30 (18). A significant decrease of blood pressure values, TAG, blood glucose and oGTT values has been described after the use of a combination of ultrasound lipolysis and liposuction (30). And as Nasrete showed in the recent study, arterial blood pressure decreased hand in hand with the weight reduction after LVL (27).

On contrary, other authors reported no effect of large-volume liposuction on arterial blood pressure in both type II diabetic and non-diabetic women with abdominal obesity as long as 3 months 
postoperatively (10). The different paper again showed a lack of effect of LVL on blood pressure values in obese patients 10 weeks to several years postoperatively (19).

This supports the theory that proinflammatory cytokines production is higher in visceral fat than in subcutaneous fat while adiponectine production behaves in the very opposite way (31). Therefore, reducing the amount of subcutaneous fat simply isn't sufficient for compensating the endothelial dysfunction resulting from excessive abdominal fat deposits and leading to arterial hypertension.

\section{Inflammatory markers}

A significant decrease of plasma TNF- $\alpha$ and IL- 6 has been reported in patients 2 months after abdominal lipectomy (abdominoplasty) when compared to the control group of patients on hypocaloric diet (32). D'Andrea et al came to similar conclusions in patients 3 months post LVL (25) as well as the Giugliano's team, who noticed a decrease in IL-6, IL-18, TNF- $\alpha$ and CRP 6 months post LVL in obese females (17). Swanson has just recently presented the paper showing a significant lowering of leukocyte blood count in the group of 322 patients 3 months after either ultrasound assisted liposuction, abdominoplasty alone or a combination of the two abovementioned procedures (9).

The more frequently performed small-volume liposuction (regular volume liposuction) presented with an immediate postoperative increase of IL- 6 and angiotensin II and decrease of adiponectin, leptin and TNF- $\alpha$ levels, but showed no effect on interleukin 6 or other adipocytokines already one month after the procedure (33).

In the 4 month follow-up, liposuction didn't influence neither CRP values (15) nor other inflammatory markers such as TNF- $\alpha$ or other adipocytokines $(10,29)$. The absence of a postoperative effect on adipsin (D factor) in blood, despite a slight intraoperative increase, seems positive as there was no excessive alternative pathway complement activation (16).

\section{Conclusion}

As the review showed, the impact of liposuction, as one of the most frequent procedures in cosmetic surgery (the most common aesthetic surgical technique in the USA), on the inception and development of metabolic syndrome, as an epidemiologically extremely important disease, has been so far studied without coming to a definite conclusion. Results that can be found in the published literature remain controversial and often contradictory, thus leaving enough space for further investigations regarding the relation of these two clinical entities.

\section{References}

1. Gingrass MK. Liposuction. 533-541. In: Thorne ChH (Ed). Grabb and Smith's Plastic Surgery. 6th ed. Philadelphia; Lippincott-Raven Publishers 2007.

2. Klein S, Fontana L, Young VL, Coggan AR, Kilo C, Patterson BW, Mohammed BS. Absence of an effect of liposuction on insulin action and risk factors for coronary heart disease. NEJM 2004; 350 (25): 2549-2557.
3. Reaven GM. Metabolic syndrome: Pathophysiology and Implications for Management of Cardiovascular diseases. Circulation 2002; 106: 286-288.

4. Miranda PJ, DeFronzo RA, Califf RM, Guyton JR. Metabolic syndrome: definition, pathophysiology, and mechanisms. Am Heart J 2005; 149: 33-45.

5. Vandeweyer et al. Does liposuction influence lipidogram in females: in vivo study. Aesth Plast Surg 2002; 26 (1): 17-19.

6. Robles-Cervantes JA, Yánez-Diaz S, Cárdenas-Camarena L. Modification of insulin, glucose and cholesterol levels in nonobese women undergoing liposuction: is liposuction metabolically safe? Ann Plast Surg 2004; 52 (1): 64-67.

7. Ma GE, Liu P, Chen J, Shi L, Cheng T, Lu W, Zhao ZM. Effect of liposuction on insulin sensitivity and lipid metabolism. Zhonghua Zheng Xing Wai Ke Za Zhi 2007; 23 (4): 313-314.

8. Montoya T, Monereo S, Olivar J, Iglesias P, Diaz P. Effects of orlistat on visceral fat after liposuction. Dermatol Surg 2009; 35 (3): 469-474.

9. Swanson E. Prospective clinical study reveals significant reduction in triglyceride level and white blood cell count after liposuction and abdominoplasty and no change in cholesterol levels. Plast Reconstr Surg 2011; 128 (3): 182-197.

10. Klein S, Fontana L, Young VL, Coggan AR, Kilo C, Patterson BW, Mohammed BS. Absence of an effect of liposuction on insulin action and risk factors for coronary heart disease. NEJM 2004; 350 (25): 2549-2557.

11. Palmieri B. Metabolic and clinical studies of liposuction in obesity. 435-438. In: Shiffman MA, Di Giuseppe A (Eds). Liposuction. Principles and practice. Berlin; Springer 2006.

12. Uwaifo G, Yanovski JA. Cardiovascular risk profile improvement with large-volume liposuction. 451-459. In: Shiffman MA, Di Giuseppe A(Eds). Liposuction. Principles and practice. Berlin; Springer 2006.

13. Hong YG, Kim HT, Seo SW, Chang CH, Rhee EJ, Lee WY. Impact of large-volume liposuction on serum lipids in orientals: a pilot study. Aesth Plast Surg 2006; 30 (3): 327-332.

14. Pedroza LV. Biochemical effects of liposuction. 15-16. In: Shiffman MA, Di Giuseppe A (eds.). Liposuction. Principles and practice. Berlin; Springer 2006.

15. Ybarra J, Blanco-Vaca F, Fernandez S, Castellvi A, Bonet R, Palomer X, Ordonez-Llanos J, Trius A, Vila-Rovira R, Perez A. The effects of liposuction removal of subcutaneous abdominal fat on lipid metabolism are independent of insulin sensitivity in normal-overweight individuals. Obes Surg 2008; 18 (4): 408-414.

16. Samdal F, Birkeland KI, Ose L, Amland PF. Effect of large-volume liposuction on sex hormones and glucose- and lipid metabolism in females. Aesthetic Plast Surg 1995; 19: 131-135.

17. Giugliano G, Nicoletti G, Grella E, Giugliano F, Esposito K, Scuderi N, D'Andrea F. Effect of liposuction on insulin resistance and vascular inflammatory markers in obese women. Br J Plast Surg 2004; 57 (3): 190-194.

18. Giese SY, Bulan EJ, Commons GW, Spear SL, Yanovski JA. Improvements in cardiovascular risk profile with large-volume liposuction: a pilot study. Plast Reconstr Surg 2001; 108: 510-519.

19. Mohammed SB, Cohen S, Reeds D, Young LV, Klein S. Long-term effects of large-volume liposuction on metabolic risk factors for coronary heart disease. Obes 2008; 16 (12): 2648-2651.

20. Hildreth B. Liposuction and serum lipids. Am J Cosm Surg 1997; 14: $345-346$. 
$349-352$

21. Palmieri B, Bosio P, Catania N, Gozzi G. La liposcultu-ra con ultrasuoni: un trattamento miniinvasivo della Obesità. Rec Progr Med 1995; 86: $220-225$.

22. Matarasso A, Kim RW, Kral JG. The impact of liposuction on body fat. Plast Reconstr Surg 1998; 102 (5): 1686-1689.

23. Hernandez TL, Kittelson JM, Law CK, Ketch LL, Stob NR, Lindstrom RC, Scherzinger A, Stamm ER, Eckel RH. Fat redistribution following suction lipectomy: defense of body fat and patterns of restoration. Obes $2011 ; 19$ (7): 1388-1395.

24. Benatti F, Solis M, Artioli G et al. Liposuction induces a compensatory increase of visceral fat which is effectively counteracted by physical activity: a randomized trial. J Clin Endocrinol Metab 2012; 97 (7): 2388-2395.

25. D'Andrea F, Grella R, Rizzo MR, Grella E, Grella R, Nicoletti G, Barbieri M, Paolisso G. Changing the metabolic profile by large-volume liposuction: a clinical study conducted with 123 obese women. Aesth Plast Surg 2005; 29 (6): 472-478.

26. Metabolic modulation by lipoplasty: a case report and invitation for investigators. Aesth Plast Surg 2004; 28 (2): 120-122.

27. Narsete T, Narsete M, Buckspan R, Ersek R. Large-volume liposuction and prevention of type 2 diabetes: a preliminary report. Aesth Plast Surg 2012; 36 (2): 438-442.
28. Cazes L, Deitel M, Levine RH. Effect of abdominal lipectomy on lipid profile, glucose handling and blood pressure in patients with truncal obesity. Obes Surg 1996; 6: 159.

29. Robles-Cervantes JA, Martinez-Abundis E, Gonzales-Ortiz M, Cardenas-Camarena L, Hernandez-Salazar E, Olvera-Ozuna R. Behavior of insulin sensitivity and its relation to leptin and tumor necrosis factor-alpha in obese women undergoing liposuction: 6-month follow-up. Obes Surg 2007; 17 (9): 1242-1247.

30. Palmieri B, Bosio P, Palmieri G, Gozzi G. Ultrasound lipolysis and suction lipectomy for treatment of obesity. Am J Cosm Surg 1997; 14: 289-296.

31. Hamdy O, Porramatikul S, Al-Ozairi E. Metabolic obesity: the paradox between visceral and subcutaneous fat. Curr Diab Rev 2006; 2 (4): 367-373.

32. Marfella R, Grella R, Rizzo MR, Barbieri M, Grella R, Ferraraccio F, Cacciapuoti F, Mazzarella G, Ferraro N, D'Andrea F, Paolisso G, Nicoletti G. Role of subcutaneous abdominal fat on cardiac function and proinflammatory cytokines in premenopausal obese women. Ann Plast Surg 2009; 63 (5): 490-495.

33. Davis DA, Pellowski DM, Davis DA, Donahoo WT. Acute and 1-month effect of small-volume suction lipectomy on insulin sensitivity and cardiovascular risk. Int J Obes 2006; 32 (8): 1217-1222.

Received September 4, 2012. Accepted January 27, 2013. 